\section{Cetoacidosis por estrés: caso clínico en paciente con atrofia muscular espinal}

\author{
CAROLINA AGUILAR ${ }^{1}$, RODRIGO ANDRÉS SEPÚLVEDA², \\ RODRIGO TAGLE ${ }^{2}$
}

\section{Stress induced ketoacidosis in spinal muscular atrophy. Report of one case}

Spinal muscular atrophy is an uncommon cause of ketoacidosis, where there is a decrease in muscle mass, an abnormal metabolism of glucose and fatty acids, and changes in neuroendocrine function. These conditions favor the accumulation of keto acids and the development of metabolic acidosis. We report a 26-year-old female, with a history of spinal muscular atrophy type III, consulting for abdominal pain and vomiting lasting one week. She was admitted to the emergency service somnolent and poorly perfused. She had a $p H$ of 6.98, $\mathrm{HCO} 3$ - of $3.8 \mathrm{mmol} / \mathrm{L}, \mathrm{pCO}$ of $16.4 \mathrm{mmHg}, \mathrm{BE}$ of $-26 \mathrm{mmol} / \mathrm{L}$, delta ratio of 1.05 , anion gap of $31 \mathrm{mEq} / \mathrm{L}$, creatinine of $0.37 \mathrm{mg} / \mathrm{dL}$, sodium of $147 \mathrm{mEq} / \mathrm{L}$, potassium of $3.7 \mathrm{mEq} / \mathrm{L}$, chloride of $112 \mathrm{mEq} / \mathrm{L}$, lactate of $1.2 \mathrm{mmol} / \mathrm{L}$, glucose of $108 \mathrm{mg} / \mathrm{dL}$, albumin of $4.2 \mathrm{~g} / \mathrm{dL}$, ketonemia +++ , ketonuria + , measured plasma osmolality of $322 \mathrm{mOsm} / \mathrm{kg}$, estimated osmolality of $314 \mathrm{mOsm} / \mathrm{kg}$, toxilab negative, salicylate levels $<3 \mu \mathrm{g} / \mathrm{mL}$, acetaminophen levels $<1.2 \mu \mathrm{g} /$ $m L$. Intravenous hydration and bicarbonate were started, without satisfactory response. Interpreting the clinical picture as a ketoacidosis induced by stress in a patient with spinal muscular atrophy, it was handled with glucose, amino acids, vitamins and trace elements, with a favorable response.

(Rev Med Chile 2020; 148: 875-880)

Key words: Acidosis; Ketosis; Muscular Atrophy, Spinal; Oxaloacetates.
${ }^{1}$ Nefrología. Especialidades Médicas de Adultos. Clínica Bupa Santiago, Chile. 2Departamento de Nefrología, Escuela de Medicina, Facultad de Medicina, Pontificia Universidad Católica de Chile. Santiago, Chile.

Trabajo no recibió financiamiento. Los autores declaran no tener conflictos de interés.

Recibido el 14 de agosto de 2019, aceptado el 8 de junio de 2020.

Correspondencia a:

Dr. Rodrigo Tagle Vargas Departamento de Nefrología. Diagonal Paraguay 362, piso 4. Santiago, Chile.

rtagle@med.puc.cl la uando se habla de cetoacidosis (CA), la mayor parte de los clínicos tiende a pensar en este cuadro como una entidad exclusiva de la diabetes mellitus (DM), más aun con la introducción de los inhibidores de SGLT2. Sin embargo, existen múltiples causas de CA no asociadas a la DM, tales como el ayuno, alcohol, fármacos, intoxicaciones, estrés y errores congénitos del metabolismo (Tabla 1$)^{1}$, que debemos tener en consideración al momento de evaluar a un paciente con acidosis metabólica.

La atrofia muscular espinal (AME) es una enfermedad neurodegenerativa que afecta motoneuronas del asta anterior de la médula espinal generando denervación y atrofia musculoesquelética. Se produce por una mutación en los genes
SMN1 y SMN2 del cromosoma 5. El espectro clínico de la AME es bastante amplio y se clasifica de acuerdo al grado de debilidad muscular, edad de inicio y pruebas genéticas (Tabla 2) ${ }^{2-4}$. La AME tipo III (enfermedad de Kugelberg-Welander) se caracteriza por iniciarse luego de los 18 meses de vida y se presenta con debilidad muscular, hipotonía y atrofia muscular, logrando una marcha autónoma, pero fallecen en la vida adulta.

La AME es una causa infrecuente de cetoacidosis, donde la disminución de masa muscular, metabolismo anormal de glucosa y ácidos grasos, alteraciones en la función pancreática y cambios en la actividad neuroendocrina, favorecen la acumulación de cetoácidos y desarrollo de una acidosis metabólica $^{4-10}$. 


\section{Tabla 1. Causas de cetosis y cetoacidosis}

\begin{tabular}{|c|c|}
\hline Diabetes mellitus & Cetoacidosis diabética \\
\hline Nutricionales & $\begin{array}{l}\text { Ayuno } \\
\text { Dietas cetogénicas }\end{array}$ \\
\hline $\begin{array}{l}\text { Aumento hormonas } \\
\text { de contrarregulación }\end{array}$ & $\begin{array}{l}\text { Estrés (infección, infarto, inflamación, isquemia, etc). } \\
\text { Cocaína } \\
\text { Éxtasis (3,4-methylenedioxymethamphetamine) }\end{array}$ \\
\hline Fármacos & $\begin{array}{l}\text { Inhibidores SGLT2 } \\
\text { Antipsicóticos atípicos (clozapina, risperidona, olanzapina, quetiapina, ziprasidona, } \\
\text { aripiprazol) } \\
\text { Isoniazida }\end{array}$ \\
\hline Intoxicaciones & $\begin{array}{l}\text { Etanol (cetoacidosis alcohólica) } \\
\text { Isopropanol* } \\
\text { Salicilatos } \\
\text { Acetona* }\end{array}$ \\
\hline $\begin{array}{l}\text { Errores congénitos del } \\
\text { metabolismo }\end{array}$ & $\begin{array}{l}\text { Acidemias orgánicas (isovalérica, propiónica, metilmalónica) } \\
\text { Déficit múltiple de carboxilasas } \\
\text { Enfermedad de orina con olor a jarabe de arce } \\
\text { Trastornos mitocondriales } \\
\text { Deficiencias de cetólisis }\end{array}$ \\
\hline
\end{tabular}

*Producen cetosis sin cetoacidosis.

Tabla 2. Subtipos de AME

\begin{tabular}{|c|c|c|c|c|}
\hline & AME tipo I & AME tipo II & AME tipo III & AME tipo IV \\
\hline $\begin{array}{l}\text { Edad de } \\
\text { comienzo }\end{array}$ & Antenatal $-<6$ meses & 7-18 meses & 18 meses - 30 años & $>30$ años \\
\hline $\begin{array}{l}\text { Hallazgos } \\
\text { motores }\end{array}$ & $\begin{array}{l}\text { No se sienta } \\
\text { Hipomotilidad } \\
\text { Sin control de mo- } \\
\text { vimientos cefálicos, } \\
\text { expresión facial normal, } \\
\text { alteración deglución, } \\
\text { respiración paradójica } \\
\text { Fasciculaciones linguales }\end{array}$ & $\begin{array}{l}\text { Sí se sienta, pero no } \\
\text { camina } \\
\text { Escoliosis } \\
\text { Contracturas articulares } \\
\text { Temblor postural en } \\
\text { manos } \\
\text { Fasciculaciones linguales } \\
\text { y/o músculos extremi- } \\
\text { dades }\end{array}$ & $\begin{array}{l}\text { Camina } \\
\text { Escoliosis } \\
\text { Contracturas articulares } \\
\text { Temblor postural en } \\
\text { manos } \\
\text { Fasciculaciones } \\
\text { músculos extremidades } \\
\text { Algunos requieren silla } \\
\text { de ruedas y/o VNI }\end{array}$ & $\begin{array}{l}\text { Desarrollo motor normal } \\
\text { en la lactancia } \\
\text { Algunos requieren } \\
\text { silla de ruedas y/o VNI } \\
\text { (ventilación mecánica } \\
\text { no invasiva) }\end{array}$ \\
\hline $\begin{array}{l}\text { Expectativa } \\
\text { de vida }\end{array}$ & $\begin{array}{l}\text { Sobrevida }<6 \text { meses } \\
\text { Muerte por insuficiencia } \\
\text { respiratoria }\end{array}$ & $\begin{array}{l}\text { Fallecen antes de } 30-40 \\
\text { años por insuficiencia } \\
\text { respiratoria }\end{array}$ & Fallecen en edad adulta & Normal \\
\hline
\end{tabular}

\section{Caso clínico}

Mujer de 26 años de edad, con antecedente de AME tipo III y escoliosis operada en la infancia. Talla $1,5 \mathrm{~m}$, peso $42 \mathrm{~kg}$, índice de masa corporal $18,7 \mathrm{~kg} / \mathrm{m}^{2}$. Funciones cognitivas normales, pero grave atrofia de musculatura axial y apendicular que la obliga a movilizarse en silla de ruedas y depender de un tercero para realizar algunas acti- vidades de vida diaria. Usuaria de anticonceptivos orales, no refiere consumo de otros fármacos.

Consulta por cuadro de una semana de evolución caracterizado por compromiso del estado general, dolor abdominal y vómitos alimentarios. Ingresa al servicio de urgencia somnolienta, normotensa 115/78 mmHg, taquicárdica $140 \mathrm{lpm}$, polipneica $30 \mathrm{rpm}$, afebril. Se realizan exámenes de ingreso destacando en niveles plasmáticos: 
$\mathrm{pH} 6,98 ; \mathrm{HCO}_{3}^{-} 3,8 \mathrm{mmol} / \mathrm{L} ; \mathrm{pCO}_{2} 16,4 \mathrm{mmHg}$; $\mathrm{EB}-26 \mathrm{mmol} / \mathrm{L}$; delta ratio 1,05 ; gap de aniones $31 \mathrm{mEq} / \mathrm{L}$; creatinina $0,37 \mathrm{mg} / \mathrm{dL}$; BUN $38 \mathrm{mg} / \mathrm{dL}$; sodio $147 \mathrm{mEq} / \mathrm{L}$; potasio $3,7 \mathrm{mEq} / \mathrm{L}$; cloro 112 $\mathrm{mEq} / \mathrm{L}$; lactato $1,2 \mathrm{mmol} / \mathrm{L}$; glucosa $108 \mathrm{mg} / \mathrm{dL}$; albúmina 4,2 g/dL; CK total $243 \mathrm{U} / \mathrm{L}$; cetonemia +++ , cetonuria + , osmolalidad plasmática medida $322 \mathrm{mOsm} / \mathrm{kg}$, osmolalidad calculada $314 \mathrm{mOsm} /$ $\mathrm{kg}$, estudios toxicológicos negativo, niveles de salicilato $<3 \mu \mathrm{g} / \mathrm{mL}$, niveles acetoaminofeno $<1,2 \mu \mathrm{g} /$ $\mathrm{mL}$. Además, tomografía computada de abdomen y pelvis (por antecedente de dolor abdominal), que mostraba nefrolitiasis bilateral y atrofia muscular toracoabdominal, sin otros hallazgos.

Fue ingresada con diagnóstico de acidosis metabólica en estudio, se inició volemización y aporte de bicarbonato endovenoso, sin respuesta satisfactoria. Se interpretó cuadro como CA inducida por estrés en paciente con AME, cuyo tratamiento es reposición de carbohidratos, evitar hipoglicemia y manejo de enfermedades intercurrentes. Se administró suero glucosado $30 \%$, aminoácidos $10 \%$, vitaminas y oligoelementos, con respuesta favorable. Gases de control pH 7,53; $\mathrm{HCO}_{3}^{-} 20,8$ $\mathrm{mmol} / \mathrm{L}$; gap de aniones $14 \mathrm{mEq} / \mathrm{L}$, siendo dada de alta a los 4 días desde su ingreso.

\section{Discusión}

Todos los organismos requieren energía para el funcionamiento celular. Evolutivamente, esta energía se obtiene desde "ácidos orgánicos". Normalmente, catabolizamos glucosa en ácido pirúvico que posteriormente entregará moléculas de
ATP. El ácido láctico, ácido cítrico, aminoácidos y ácidos grasos también son fuentes energéticas importantes para las células. Los ácidos orgánicos permiten generar moléculas de alto poder reductor $\left(\mathrm{NADH}_{\text {y }} \mathrm{FADH}_{2}\right)$ que en la mitocondria aportarán el gradiente de $\mathrm{H}^{+}$necesario para la síntesis de $\mathrm{ATP}^{11}$. Los cetoácidos cumplen la misma función anteriormente mencionada, sin embargo, no todas las células pueden metabolizarlos. Solo el corazón, riñón, músculo y cerebro poseen la maquinaria enzimática que les permite utilizar cetoácidos como fuente energética ${ }^{12}$. Dado que el tejido muscular representa $\approx 40 \%$ del peso corporal, es el parénquima que metaboliza la mayor cantidad de cetoácidos, tanto en reposo como en ejercicio ${ }^{13}$. Si existe sobreproducción de cetoácidos y los parénquimas antes mencionados no los metabolizan, sobrevendrá una cetoacidosis.

Cuando ocurre una condición de estrés o ausencia de insulina, como en la DM, la mayor movilización y catabolización de ácidos grasos, sumado a una falta de inhibición en la cetogénesis, favorecerán síntesis exagerada de ácido acetoacético y ácido $\beta$-hidroxibutírico, provocando acidosis metabólica ${ }^{14}$.

Otro mecanismo que promueve sobreproducción de cetoácidos es la depleción de oxaloacetato intracelular. El oxaloacetato es necesario para evitar la formación de cetoácidos y también es fundamental en la gluconeogénesis ${ }^{15}$. Si todo el oxaloacetato es utilizado en gluconeogénesis (como ocurre en estados de ayuno) no existirá disponibilidad de este para evitar la formación de cetoácidos (Figura 1).

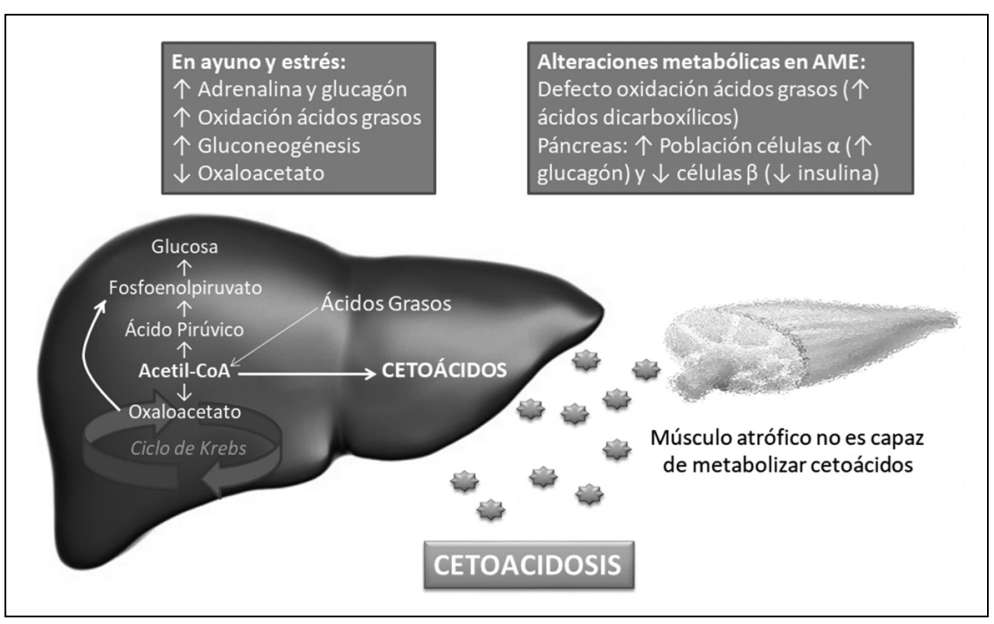

Figura 1. Mecanismo de cetoacidosis en atrofia muscular espinal. 
El consumo de etanol y los estados de estrés se asocian a una activación de hormonas de contrarregulación, inhibición de insulina, más un estado de ayuno (activación de gluconeogénesis). El uso de inhibidores SGLT2 provoca pérdida masiva de glucosa en la orina, lo que disminuye la secreción de insulina, aumenta las hormonas de contrarregulación, activa la gluconeogénesis y produce movilización de ácidos grasos para obtener energía ${ }^{16}$. Estas condiciones favorecen cetogénesis. A diferencia de la intoxicación por paracetamol (que induce acidosis piroglutámica), en la intoxicación por salicilatos también existe una movilización masiva de ácidos grasos con disfunción mitocondrial que propician una cetoacidosis ${ }^{17}$.

En toda situación de estrés o ayuno habrá cetogénesis. En la AME, el tejido muscular atrófico no puede utilizar cetoácidos para obtener energía, favoreciendo su acumulación y desarrollo de acidosis metabólica. De esta forma, se configura la CA inducida por estrés, que siendo una causa rara de CA debe ser sospechada en todo paciente con AME o alguna patología que impida la utilización de cetoácidos como fuente energética.

Nuestra paciente se presentó con un cuadro grave de acidosis metabólica con anion gap aumentado. No presentaba alteración en la perfusión tisular, función renal u osmolalidad que explicaran este cuadro. No hubo consumo de paracetamol ni de salicilatos. La prueba de cetonuria resultó muy positiva, confirmando un cuadro de cetoacidosis. Es probable que en la acidosis también participara la acumulación de ácidos dicarboxílicos ${ }^{6}$. Presumimos que el factor desencadenante fue el estrés asociado a un cuadro gastrointestinal, sumándose, posteriormente, un estado de ayuno y deshidratación.

En estados de estrés, las células presentan un mayor potencial oxidativo que favorece la oxidación de distintas moléculas. Lo anterior produce que gran parte del ácido acetoacético sea transformado en ácido $\beta$-hidroxibutírico. Este último no es una cetona, por lo que no es detectado en las reacciones semicuantitativas con nitroprusiato (cetonuria y cetonemia), en consecuencia, no se pesquisa con las técnicas de laboratorio tradicionales ${ }^{1,14,18}$. Es así como, en CA diabética y alcohólica, puede haber un resultado falso negativo con estos exámenes. Por lo anterior, es recomendable medir directamente niveles de $\beta$-hidroxibutirato, sin embargo, en las cetoacidosis producidas por errores congénitos del metabolismo, no se acumulan ácidos acetoacético, ni $\beta$-hidroxibutírico, de manera que se debe medir cetonuria y cetonemia, aunque tengan menor sensibilidad.

El delta ratio de la paciente fue 1,05, compatible con una acidosis metabólica con anion gap aumentado. Sin embargo, se encuentra en rango bajo, porque la CA es una combinación de acidosis metabólica con gap de aniones aumentado más hiperclorémica ${ }^{1920}$. Es destacable que la compensación respiratoria haya sido adecuada en una paciente que presenta atrofia muscular. Esto se explica porque la musculatura que se utiliza regularmente o se ejercita frecuentemente, desarrolla enzimas capaces de realizar cetólisis ${ }^{13}$. Por lo tanto, la musculatura respiratoria se vio favorecida del exceso de cetoácidos.

El tratamiento de la CA requiere controlar el factor causal e inhibir la sobreproducción de cetoácidos. Para bloquear la cetogénesis basta con reponer el oxaloacetato intracelular, condición que se logra al administrar glucosa intravenosa, inhibiendo la gluconeogénesis. Por lo tanto, el tratamiento es simple y efectivo para CA inducida por estrés, ayuno, etanol y fármacos. En nuestra paciente se realizó una infusión de glucosa que rápidamente permitió solucionar el cuadro. La insulina puede ser perjudicial en CA no diabética, ya que normalmente existe un estado de normo o hipoglicemia sostenido por la intensa gluconeogénesis. La insulina podría precipitar hipoglicemias con riesgo vital. El bicarbonato de sodio intravenoso es controversial en pacientes con cetoacidosis ${ }^{21}$, pero cuando el cuadro es grave, puede ser necesario para evitar las importantes consecuencias cardiovasculares de la acidemia ${ }^{22}$. La infusión inicial de bicarbonato no tuvo ningún efecto en nuestra paciente, sí, en cambio, la infusión de glucosa y aminoácidos (Figura 2).

En conclusión, presentamos un caso de CA no diabética, en donde una condición de estrés y ayuno, sumado a la atrofia muscular producto de AME, producen una acidosis metabólica grave con riesgo vital. La identificación de este tipo de CA por parte de médicos de urgencia e intensivistas es fundamental, ya que su tratamiento es muy simple y un error diagnóstico o tratamiento inadecuado puede aumentar la morbilidad del paciente. 


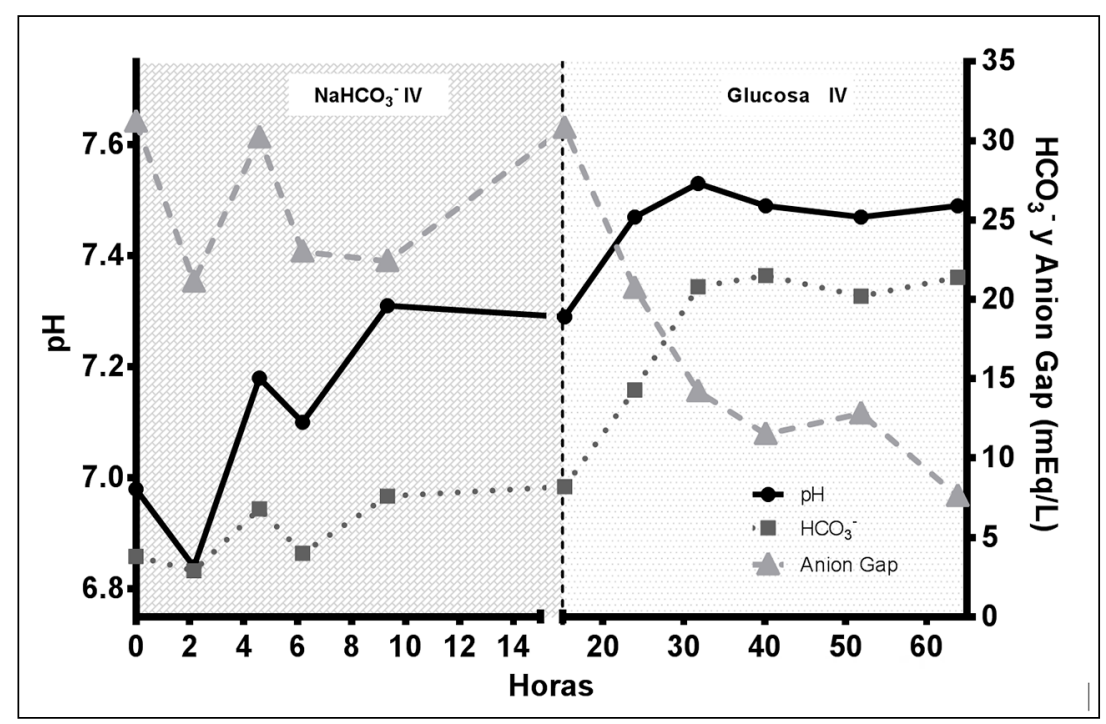

Figura 2. Evolución clínica parámetros ácido-base.

\section{Referencias}

1. Cartwright MM, Hajja W, Al-Khatib S, Hazeghazam M, Sreedhar D, Li RN, et al. Toxigenic and metabolic causes of ketosis and ketoacidotic syndromes. Crit Care Clin 2012; 28 (4): 601-31.

2. Wirth B. An update of the mutation spectrum of the survival motor neuron gene (SMN1) in autosomal recessive spinal muscular atrophy (SMA). Hum Mutat 2000; 15 (3): 228-37.

3. Castiglioni C, Levicán J, Rodillo E, Garmendia MA, Díaz A, Pizarro L, et al. [Clinical, electrophysiological and molecular study of 26 chilean patients with spinal muscular atrophy]. Rev Med Chile 2011; 139 (2): 197-204.

4. Shababi M, Lorson CL, Rudnik-Schöneborn SS. Spinal muscular atrophy: a motor neuron disorder or a multi-organ disease? J Anat 2014; 224 (1): 15-28.

5. Zolkipli Z, Sherlock M, Biggar WD, Taylor G, Hutchison JS, Peliowski A, et al. Abnormal fatty acid metabolism in spinal muscular atrophy may predispose to perioperative risks. Eur J Paediatr Neurol 2012; 16 (5): 549-53.

6. Kelley RI, Sladky JT. Dicarboxylic aciduria in an infant with spinal muscular atrophy. Ann Neurol 1986; 20 (6): 734-6.

7. Lakkis B, El Chediak A, Hashash JG, Koubar SH. Severe ketoacidosis in a patient with spinal muscular atrophy. CEN Case Rep 2018;7 (2): 292-5.

8. Mulroy E, Gleeson S, Furlong MJ. Stress-Induced Ketoacidosis in Spinal Muscular Atrophy: An Under-Re- cognized Complication. J Neuromuscul Dis 2016; 3 (3): 419-23.

9. Stoimenis D, Spyridonidou C, Theofanidou S, Petridis $\mathrm{N}$, Papaioannou N, Iasonidou C et al. Euglycemic Ketoacidosis in Spinal Muscular Atrophy. Case Rep Pediatr 2019; 2019: 2862916.

10. Bowerman M, Swoboda KJ, Michalski JP, Wang GS, Reeks C, Beauvais A et al. Glucose metabolism and pancreatic defects in spinal muscular atrophy. Ann Neurol 2012; 72 (2): 256-68.

11. Wallace KB, Starkov AA. Mitochondrial targets of drug toxicity. Annu Rev Pharmacol Toxicol 2000; 40: 35388.

12. Robinson AM, Williamson DH. Physiological roles of ketone bodies as substrates and signals in mammalian tissues. Physiol Rev 1980; 60 (1): 143-87.

13. Evans M, Cogan KE, Egan B. Metabolism of ketone bodies during exercise and training: physiological basis for exogenous supplementation. J Physiol 2017; 595 (9): 2857-71.

14. Laffel L. Ketone bodies: a review of physiology, pathophysiology and application of monitoring to diabetes. Diabetes Metab Res Rev 1999; 15 (6): 412-26.

15. Berg JM, Tymoczko JL, Stryer L. Glycolysis and Gluconeogenesis. En: Berg JM, Tymoczko JL, Stryer L, Editores, Biochemistry 5th edition. New York, USA: Editorial W H Freeman; 2002. p. 1631-37.

16. Yu X, Zhang S, Zhang L. Newer Perspectives of Mechanisms for Euglycemic Diabetic Ketoacidosis. Int J Endocrinol 2018; 2018: 7074868. 
17. Sepúlveda RA, Ortega M, Donoso N, Jara A. [Physiopathology and management of acetylsalicylic acid intoxication]. Rev Med Chile 2018; 146 (11): 1309-16.

18. Dhatariya K. Blood Ketones: Measurement, Interpretation, Limitations, and Utility in the Management of Diabetic Ketoacidosis. Rev Diabet Stud 2016; 13 (4): 217-25.

19. Adrogué HJ, Wilson H, Boyd AE 3rd, Suki WN, Eknoyan G. Plasma acid-base patterns in diabetic ketoacidosis. N Engl J Med 1982; 307 (26): 1603-10.
20. Palmer BF, Clegg DJ. Electrolyte and Acid-Base Disturbances in Patients with Diabetes Mellitus. N Engl J Med 2015; 373 (6): 548-59.

21. Patel MP, Ahmed A, Gunapalan T, Hesselbacher SE. Use of sodium bicarbonate and blood gas monitoring in diabetic ketoacidosis: A review. World J Diabetes 2018; 9 (11): 199-205.

22. Kraut JA, Madias NE. Treatment of acute metabolic acidosis: a pathophysiologic approach. Nat Rev Nephrol 2012; 8 (10): 589-601. 